\title{
First reported case of Multisystem Inflammatory Syndrome in children in West Texas
}

\author{
Ryan E. Dean BS, MBA, Monisha Narayanan BS, Evan Nix BS, MBA, \\ Kinsley Stepka DO, Raphael Mattamal, MD
}

\begin{abstract}
Research focusing on COVID-19-associated complications is a growing area of importance. One such complication is multisystem inflammatory syndrome, a rare immune-mediated condition that most commonly presents with Kawasaki-like symptoms in the pediatric population. Potential complications include myocarditis, renal impairment, and cytokine storm. Here we describe the first reported case of multisystem inflammatory syndrome in the West Texas region, presenting in a Hispanic 5-year-old girl with a recent history of COVID-19. The patient arrived to the hospital with a 4-day history of high fever, a 2-day history of diffuse maculopapular rash, and complaints of fatigue, generalized body aches, decreased appetite, headache, and abdominal pain. Further physical examination revealed hepatosplenomegaly and cervical lymphadenopathy; lab tests revealed elevated inflammatory markers, lymphopenia, left shift with bandemia and immature cells, thrombocytopenia, hypoalbuminemia, and transaminitis. The patient was admitted to the pediatric intensive care unit with a suspected Kawasaki-like illness and started on high dose aspirin and IV immunoglobulin. She was placed on methylprednisolone, albumin, and acetaminophen on hospital day 3. By hospital day 4, the patient defervesced, inflammatory markers decreased, and clinical symptoms improved. The patient was discharged on hospital day 10 with absent fever and improvement of clinical symptoms for 6 consecutive days. No complications were detected upon follow-up 2 weeks later. A low threshold of suspicion for this illness is required in any child with a history of SARS-CoV-2 infection, as the presentation is vague and early identification is necessary to prevent further complications.
\end{abstract}

Keywords: COVID-19, pediatric, coronavirus, children, MIS-C

\section{INTRODUCTION}

The COVID-19 pandemic has brought unpredictable dangers to all demographics within the healthcare system. While the pediatric population represents $8.1 \%$ of all US COVID- 19 cases and $<0.1 \%$ of deaths, a new and alarming complication has occurred in children known as the multisystem inflammatory syndrome

Corresponding author: Evan Nix

Contact Information: Evan.Nix@ttuhsc.edu

DOI: 10.12746/swrccc.v9i37.779
(MIS-C). ${ }^{1}$ This case describes a patient who developed a Kawasaki-like illness 4 weeks after being diagnosed with COVID-19. To our knowledge, this is the first reported case of MIS-C in the West Texas region.

\section{Case Summary}

A previously healthy Hispanic 5-year-old girl presented to the hospital with fever, headache, and abdominal pain and subsequently tested positive for COVID-19. The patient had complete symptom resolution after a week and tested negative for the virus 12 days later. Two and one half weeks after testing negative, the patient 
was evaluated in the clinic for 4 days of antipyreticresistant high fevers $\left(102-103^{\circ} \mathrm{F}\right)$ and 2 days of diffuse rash. The patient also complained of fatigue, generalized body aches, abdominal pain, decreased appetite, and headache, but denied shortness of breath, cough, conjunctivitis, or changes to bowel habits. Physical examination was significant for a diffuse, blanchable, maculopapular rash, edema and desquamation of the hands and feet, bilateral cervical chain lymphadenopathy, and mild hepatosplenomegaly. Lab tests revealed significant lymphopenia, left shift with bandemia and immature cells, mild thrombocytopenia, transaminitis, and hypoalbuminemia. Mild sterile pyuria was noted on urinalysis. Inflammatory markers were significantly elevated, including C-reactive protein, erythrocyte sedimentation rate (ESR), fibrinogen, D-dimer, and lactate dehydrogenase (LDH). COVID-19 PCR nasopharyngeal swab was negative. Echocardiography, ECG, BNP, troponin, chest and abdominal x-rays, BUN, and creatinine were all within normal limits. The patient was given the diagnosis of MIS-C.

The patient was admitted to the PICU and started on IVIG and high dose aspirin. She was started on broad spectrum IV antibiotic coverage with ceftriaxone on day 1 followed by cefepime and linezolid on day 2 . Antibiotic coverage was discontinued after blood and urine cultures were confirmed negative on day 5. On day 2 , computed tomography with an angiogram was performed due to elevated D-dimer, mild intermittent hypoxia, shortness of breath, chest pain, and risk of hypercoagulability associated with MIS-C; this study revealed some mild subsegmental atelectasis but no pulmonary emboli. On day 3 , the patient was started on methylprednisolone, acetaminophen, and albumin for worsening hypoalbuminemia. The patient was also started on remdesivir, but this was discontinued 3 days later due to development of irritability and maculopapular rash unrelieved by diphenhydramine.

Throughout the hospital course, the patient continued to complain of persistent abdominal pain, fever, and recurrent episodes of nighttime hypoxia and tachypnea which required minimal oxygen support. Subsequent abdominal ultrasound showed minimal free fluid in the abdomen with mild hepatomegaly. Chest X-ray revealed pulmonary vascular congestion and bibasilar infiltrates indicative of atelectasis. The patient defervesced on day 4 and began to see significant improvement of edema, rash, abdominal pain, and atelectasis with regular physical therapy and incentive spirometry. On day 7, IV methylprednisolone was transitioned to oral prednisolone and the oral aspirin dose was reduced. On day 8 , the patient complained of new pain and swelling in the right upper extremity, and a repeat D-dimer level was elevated. Right upper extremity ultrasound revealed no thrombosis.

On day 10, after marked clinical improvement and 6 days of being afebrile, the patient was discharged with close follow up. At this time, labs had normalized except for D-dimer, erythrocyte sedimentation rate, and CRP, which continued to trend downward and platelets which continued to trend upward. Echocardiography confirmed no coronary artery abnormalities or myocardial dysfunction at pediatric cardiology follow up 2 weeks later, and, after 6 weeks of treatment, aspirin was discontinued.

\section{Discussion}

Multisystem inflammatory syndrome is a rare and emerging condition, with only 2 individuals per 100,000 under the age of 21 diagnosed with the syndrome. ${ }^{2}$ Available data have yet to determine why some children with COVID-19 develop this complication while others recover without sequelae. Despite the fact that pediatric COVID-19 is most severe in infants under 1 year of age, MIS-C most often affects older children and adolescents without comorbidities. ${ }^{3}$ Similar to COVID-19, MIS-C disproportionately affects AfricanAmerican and Hispanic children. ${ }^{2}$

Multisystem inflammatory syndrome presents similarly to other immune-mediated pediatric diseases, such as Kawasaki disease (KD) and toxic shock syndrome. Recently, Pouletty et al. published a case series comparing the presentation of MIS-C and KD and concluded that MIS-C should be suspected in any pediatric patient with an unexplained persistent high-grade fever and an elevated CRP after testing positive for SARS-CoV-2 within 4 weeks of symptom onset. In these patients, troponin, ECG, inflammatory markers, and creatinine should be obtained promptly to rule out severe complications such 
as myocarditis, cytokine storm, and renal impairment. ${ }^{4}$ Concomitantly, a CBC with differential, CMP, urinalysis, blood culture, inflammatory markers, chest x-ray, and echocardiogram should be obtained. Throughout the course of the hospital stay, inflammatory markers such as D-dimer, ESR, and CRP should be recorded daily to trend over time. ${ }^{5}$

Comparing symptoms in those with MIS-C and $K D$, children with MIS-C were significantly more likely to have gastrointestinal symptoms and myocarditis, especially those with severe MIS-C. Also, patients with MIS-C often experienced a cytokine storm, presenting with heart failure, pneumonia, gastrointestinal symptoms, and/or neurologic symptoms in the presence of elevated inflammatory markers. ${ }^{4}$ Unlike KD, MIS-C can present with thrombocytopenia, lymphocytopenia, and elevated PT/PTT, troponin, and D-dimer. ${ }^{6}$ In our patient, the development of lymphocytopenia, thrombocytopenia, and elevation of inflammatory markers such as fibrinogen and D-dimer following COVID-19 infection, along with hematologic, gastrointestinal, and dermatologic involvement on presentation made MIS-C the most likely diagnosis.

The goals for management of MIS-C broadly involve reducing systemic inflammation and minimizing the risk of long-term sequelae. At initial presentation, a multidisciplinary approach should be taken, with collaboration among the pediatric intensive care unit, infectious disease, hematology, and rheumatology divisions. All patients should be treated with broadspectrum antibiotics until MIS-C is the established diagnosis and bacterial cultures are negative. Similarly, IVIG is recommended in all cases of MIS-C due to its immunomodulating effects. However, because the exact pathophysiology is currently unknown, the specific treatment strategies are derived from other diseases with similar presentations. If symptoms meet the criteria for KD, then management is based on the KD guidelines. This includes high-dose IVIG and aspirin, with the addition of corticosteroids and/or anakinra for treatment-refractory disease. If there are no KD features and a cytokine storm-like presentation is more prominent, a regimen of corticosteroids and interleukin inhibitors such as anakinra or tocilizumab are currently recommended. Our patient improved with IVIG, aspirin, and corticosteroids, and IL-6 inhibitor medication was determined not to be needed. Remdesivir is currently indicated only for patients with a diagnosis of MIS-C who test RT-PCR positive for SARS-CoV-2; in this case it was used due to concern for false negative testing and potential reinfection from her parents from whom she initially was infected. ${ }^{6}$

Early identification and treatment of this syndrome is crucial to the prevention of the numerous multisystem complications of this disease. It is important to maintain a low threshold of suspicion for this illness as the presentation may be vague, but it should be on the differential for any child with current or previous history of COVID infection in the past few weeks.

Article citation: Dean RE, Narayanan M, Nix E, Stepka K, Mattamal R. The First reported case of Multisystem Inflammatory Syndrome in children in West Texas. The Southwest Respiratory and Critical Care Chronicles 2021;9(37):66-69

From: Department of Pediatrics, Texas Tech University Health Sciences Center, Amarillo, Texas

Submitted: $10 / 14 / 2020$

Accepted: $12 / 15 / 2020$

Reviewer: Adaobi Kanu MD

Conflicts of interest: none

This work is licensed under a Creative Commons Attribution-ShareAlike 4.0 International License.

\section{REFERENCES}

1. CDC COVID Data Tracker [Internet]. Atlanta (GA): Centers for Disease Control. c2020 - [cited 2020 Sept 1]. Available at: https:// covid.cdc.gov/covid-data-tracker/?utm_source=morning_ brew\#demographics

2. Dufort EM, Koumans EH, Chow EJ, et al. Multisystem inflammatory syndrome in children in New York State. N Engl J Med 2020;383(4):347-358.

3. Whittaker E, Bamford A, Kenny J, et al. Clinical characteristics of 58 children with a pediatric inflammatory multisystem syndrome temporally associated with SARS-CoV-2. JAMA 2020;324(3):259-269.

4. Pouletty M, Borocco C, Ouldali N, et al. Paediatric multisystem inflammatory syndrome temporally associated with 
SARS-CoV-2 mimicking Kawasaki disease (Kawa-COVID-19): A multicentre cohort. Ann Rheum Dis 2020;79(8):999-1006.

5. Hennon TR, Penque MD, Abdul-Aziz R, et al. COVID-19 associated multisystem inflammatory syndrome in children (MIS-C) guidelines; a Western New York approach. Prog Pediatr Cardiol 2020;57:101232.
6. Nakra NA, Blumberg DA, Herrera-Guerra A, et al. MultiSystem Inflammatory Syndrome in Children (MIS-C) following SARS-CoV-2 infection: review of clinical presentation, hypothetical pathogenesis, and proposed management. Children (Basel) 2020;7(7):69. 